\title{
ON WEAKLY 2-ABSORBING SEMI-PRIMARY SUBMODULES OF MODULES OVER COMMUTATIVE RINGS
}

\section{PAIROTE YIARAYONG* AND MANOJ SIRIPITUKDET}

Department of Mathematics, Faculty of Science, Naresuan University, Phitsanuloke 65000, Thailand

*Corresponding author: pairote0027@hotmail.com

\begin{abstract}
Let $R$ be a commutative ring with identity and let $M$ be a unitary $R$-module. We say that a proper submodule $N$ of $M$ is a weakly 2-absorbing semi-primary submodule if $a_{1}, a_{2} \in R, m \in N$ with $0 \neq a_{1} a_{2} m \in N$, then $a_{1} a_{2} \in \sqrt{(N: M)}$ or $a_{1} m \in N$ or $a_{2}^{n} m \in N$ for some positive integer $n$. In this paper, we study weakly 2-absorbing semi-primary submodules and we prove some basic properties of these submodules. Also, we give a characterization of weakly 2-absorbing semi-primary submodules and we investigate weakly 2-absorbing semi-primary submodules of some well-known modules.
\end{abstract}

\section{INTRODUCTION}

Throughout this paper, we assume that all rings are commutative with $1 \neq 0$. Let $R$ be a commutative ring and let $M$ be an $R$-module. We will denote by $(N: M)$ a residual of $N$ by $M$, that is, the set of all $r \in R$ such that $r M \subseteq N$. Clearly, $\sqrt{I}=\left\{r \in R: r^{n} \in I\right.$ for some positive integer $\left.n\right\}$ denotes the radical ideal of $R$.

In 2003, Anderson and Smith [1] introduced the concept of a weakly prime ideal of a commutative ring. They said that a proper ideal $P$ of the commutative ring $\mathrm{R}$ is weakly prime if $a, b \in R$ and $0 \neq a b \in P$, then $a \in P$ or $b \in P$. A weakly primary ideals were first introduced and studied by Atani and Farzalipour in [2]. Recall that a proper ideal $P$ of $R$ is called a weakly primary ideal of $R$ as in [2] if for $a, b \in R$ with $0 \neq a b \in P$, then $a \in P$ or $b^{n} \in P$ for some positive integer $n$. Clearly, a weakly prime ideal of $R$ is also a

2010 Mathematics Subject Classification. 13A15,13F05.

Key words and phrases. weakly 2-absorbing semi-primary submodule; 2-absorbing semi-primary submodule; absorbing semiprimary triple-zero; weakly 2-absorbing primary ideal.

(C) 2018 Authors retain the copyrights of their papers, and all open access articles are distributed under the terms of the Creative Commons Attribution License. 
weakly primary ideal of $R$. The concept of weakly 2-absorbing ideals, which is a generalization of 2-absorbing ideals, was introduced by Badawi and Darani in [3]. Recall from [3] that a proper ideal $I$ of $R$ is said to be a weakly 2-absorbing ideal of $R$ if whenever $a, b, c \in R$ with $0 \neq a b c \in I$, then $a b \in I$ or $a c \in I$ or $b c \in I$. In [4], Badawi et. al. defined a proper ideal $I$ of a commutative ring $R$ to be a weakly 2 -absorbing primary ideal if whenever $a, b, c \in R$ and $0 \neq a b c \in I$, then $a b \in I$ or $a c \in \sqrt{I}$ or $b c \in \sqrt{I}$.

The concept of weakly prime submodule was introduced and studied by Behboodi and Koohi [5]. We recall that a proper submodule $N$ of $M$ is called a weakly prime submodule, if $0 \neq r m \in N$, where $r \in R, m \in M$, then $m \in N$ or $r \in(N: M)$. The idea of decomposition of submodules into weakly primary submodules were introduced by Atani and Farzalipour in [2]. A weakly primary submodule $N$ of $M$ to be a proper submodule of $M$ and if $r \in R, m \in M$ and $0 \neq r m \in N$, then $m \in N$ or $r^{n} \in(N: M)$ for some positive integer $n$. Clearly, every primary submodule of a module is a weakly primary submodule. In [6], the concept of weakly 2-absorbing submodule generalized to 2-absorbing submodule of a module over a commutative ring. A proper submodule $N$ of $M$ is called a weakly 2-absorbing submodule, if whenever $a, b \in R$ and $m \in M$ with $0 \neq a b m \in N$, then $a b \in(N: M)$ or $a m \in N$ or $b m \in N$. In 2016, Mostafanasab et al. [11] introduced the concept of weakly 2-absorbing primary submodules of modules over commutative rings with identities. Recall that a proper submodule $N$ of $M$ is called a weakly 2-absorbing primary submodule of $M$ as in [11] if whenever $0 \neq a b m \in N$ for some $a, b \in R$ and $m \in M$, then $a b \in(N: M)$ or $a m \in M-\operatorname{rad}(N)$ or $b m \in M-\operatorname{rad}(N)$. The concept of weakly classical prime submodule, which is a generalization of classical prime submodule, was introduced by Mostafanasab et al. in [10]. Recall from [10] that a proper submodule $N$ of $M$ is said to be a weakly classical prime submodule of $M$ if whenever $a, b \in R$ and $m \in M$ with $0 \neq a b m \in N$, then $a m \in N$ or $b m \in N$. The concept of weakly classical primary submodule, a generalization of primary submodules was introduced and investigated in [9]. He weakly classical primary submodule $N$ of $M$ to be a proper submoduleof $R$ and if $a, b \in R$ and $0 \neq a b m \in N$, then $a m \in N$ or $m b^{n} \in N$ for some positive integer $n$.

Motivated and inspired by the above works, the purposes of this paper are to introduce generalizationS of weakly 2-absorbing primary submodule to the context of weakly 2-absorbing semi-primary submodule. A proper submodule $N$ of $M$ to be a weakly 2-absorbing semi-primary submodule of $M$ if whenever $0 \neq a_{1} a_{2} m \in N$ for $a_{1}, a_{2} \in R, m \in M$, then $a_{1} a_{2} \in \sqrt{(N: M)}$ or $a_{1} m \in N$ or $a_{2}^{n} m \in N$ for some positive integer $n$. Some characterizations of weakly 2-absorbing semi-primary submodules are obtained. Moreover, we investigate relationships between 2-absorbing semi-primary and weakly 2-absorbing semi-primary submodules of modules over commutative rings. 


\section{Properties of Weakly 2-Absorbing Semiprimary Submodules}

The results of the following theorems seem to play an important role to study weakly 2-absorbing semiprimary submodules of modules over commutative rings; these facts will be used frequently and normally we shall make no reference to this definition.

Definition 2.1. A proper submodule $N$ of an $R$-module $M$ is called a weakly 2-absorbing semi-primary (2-absorbing semi-primary) submodule, if for each $m \in M$ and $a_{1}, a_{2} \in R, 0 \neq a_{1} a_{2} m \in N\left(a_{1} a_{2} m \in N\right)$, then $a_{1} a_{2} \in \sqrt{(N: M)}$ or $a_{1} m \in N$ or $a_{2}^{n} m \in N$ for some positive integer $n$.

Remark 2.1. It is easy to see that every weakly 2-absorbing primary submodule (2-absorbing semi-primary) submodule is weakly 2-absorbing semi-primary submodule.

The following example shows that the converse of Definition 2.1 is not true.

Example 2.1. Let $R=\boldsymbol{Z}$ and $M=\boldsymbol{Z}$. Consider the submodule $N=12 \boldsymbol{Z}$ of $M$. It is easy to see that $N$ is a 2-absorbing semi-primary submodule of $M$. Notice that $2 \cdot 2 \cdot 3 \in N$, but $2 \cdot 3 \notin N$ and $(2 \cdot 2)^{n} \notin(N: M)$ for all positive integer $n$. Therefore $N$ is not a 2-absorbing primary submodule of $M$.

Example 2.2. Let $R=Z$ and $M=Z_{30}$. Consider the submodule $N=\{[0]\}$ of $M$. It is easy to see that $N$ is a weakly 2-absorbing semi-primary submodule of $M$. Notice that $(2 \cdot 3)[5] \in\{[0]\}$, but $2 \cdot 3 \notin \sqrt{(N: M)}, 2[5] \notin$ $\{[0]\}$ and $3^{n}[5] \notin\{[0]\}$ for all positive integer $n$. Therefore $N$ is not a 2-absorbing semi-primary submodule of $M$.

Theorem 2.1. Let $N$ be a proper submodule of an $R$-module $M$. Then the following statements hold:

(1) If $N$ is a weakly 2-absorbing semi-primary submodule of $M$, then $(N: m)$ is a weakly 2-absorbing primary ideal of $R$ for every $m \in M-N$.

(2) For every $m \in M-N$ if $(N: m)$ is a weakly primary ideal of $R$, then $N$ is a weakly 2-absorbing semi-primary submodule of $M$.

Proof. 1. Let $a_{1}, a_{2}, a_{3} \in R$ such that $0 \neq a_{1} a_{2} a_{3} \in(N: m)$. Clearly, $0 \neq a_{1} a_{3}\left(a_{2} m\right) \in N$. By Definition 2.1, $a_{1} a_{3} \in \sqrt{(N: M)} \subseteq \sqrt{(N: m)}$ or $a_{1} a_{2} m \in N$ or $a_{3}^{n} a_{2} m \in N$ for some positive integer $n$. Therefore $a_{1} a_{2} \in(N: m)$ or $a_{2} a_{3} \in \sqrt{(N: m)}$ or $a_{1} a_{3} \in \sqrt{(N: m)}$. Hence $(N: m)$ is a weakly 2-absorbing primary ideal of $R$.

2. Let $a_{1}, a_{2} \in R$ such that $0 \neq a_{1} a_{2} m \in N$. Then $0 \neq a_{1} a_{2} \in(N: m)$. By assumption, $a_{1} \in(N: m)$ or $a_{2}^{n} \in(N: m)$ for some positive integer $n$. Therefore $a_{1} m \in N$ or $a_{2}^{n} m \in N$ for some positive integer $n$. Hence $N$ is a weakly 2-absorbing semi-primary submodule of $M$.

But the converse of the above theorem is not true. For every $m \in M-N$, if $(N: m)$ is weakly 2absorbing primary ideal, then $N$ may not be weakly 2-absorbing semi-primary. Let $M=\mathbf{Z} \times \mathbf{Z} \times \mathbf{Z}$ be an 
Z-module. Consider the submodule $N=\{0\} \times 6 \mathbf{Z} \times \mathbf{Z}$ of $M$. Clearly, $\left(N:\left(m_{1}, m_{2}, m_{3}\right)\right)=\{0\}$ is a weakly 2-absorbing primary ideal of $R$, where $\left(m_{1}, m_{2}, m_{3}\right) \in M-N$. Notice that $(0,0,0) \neq(2 \cdot 3)(0,1,1) \in N$, but $2 \cdot 3 \notin \sqrt{(N: M)}, 2(0,1,1) \notin N$ and $3^{n}(0,1,1) \notin N$ for all positive integer $n$. Therefore $N$ is not a weakly 2-absorbing semi-primary submodule of $M$.

Theorem 2.2. If $N$ is a weakly 2-absorbing semi-primary submodule of an $R$-module $M$, then $(N: r)$ is a weakly 2-absorbing semi-primary submodule of $M$ containing $N$ for every $r \in R-(N: M)$.

Proof. Let $a_{1}, a_{2} \in R$ and $m \in M$ such that $0 \neq a_{1} a_{2} m \in(N: r)$. Then $0 \neq a_{1} a_{2}(r m)=r a_{1} a_{2} m \in N$. By Definition 2.1, $a_{1} a_{2} \in \sqrt{(N: M)}$ or $a_{1} r m \in N$ or $a_{2}^{n} r m \in N$ for some positive integer $n$. Therefore $a_{1} a_{2} \in \sqrt{(N: M)}$ or $a_{1} m \in(N: r)$ or $a_{2}^{n} \in(N: r)$ for some positive integer $n$. Hence $(N: r)$ is a weakly 2-absorbing semi-primary submodule of $M$.

Theorem 2.3. Let $\{0\}$ be a 2-absorbing semi-primary submodule of an $R$-module $M$. Then $N$ is a weakly 2-absorbing semi-primary submodule of $M$ if and only if $N$ is a 2-absorbing semi-primary submodule of $M$.

Proof. Suppose that $N$ is a 2-absorbing semi-primary submodule of $M$. Clearly, $N$ is a weakly 2 -absorbing semi-primary submodule of $M$.

Conversely, assume that $N$ is a weakly 2-absorbing semi-primary submodule of $M$. Let $a_{1}, a_{2} \in R$ and $m \in M$ such that $a_{1} a_{2} m \in N$. If $a_{1} a_{2} m \notin\{0\}$, then $0 \neq a_{1} a_{2} m \in N$. By Definition 2.1, $a_{1} a_{2} \in \sqrt{(N: M)}$ or $a_{1} m \in N$ or $a_{2}^{n} m \in N$ for some positive integer $n$. Now if $a_{1} a_{2} m \in\{0\}$, then $a_{1} a_{2} \in \sqrt{(N: M)}$ or $a_{1} m \in N$ or $a_{2}^{n} m \in N$ for some positive integer $n$. Hence $N$ is a 2-absorbing semi-primary submodule of M.

Theorem 2.4. Let $M$ and $M^{\prime}$ be two $R$-modules and $f: M \rightarrow M^{\prime}$ be an epimorphism of an R-module. If $N$ is a weakly 2-absorbing semi-primary submodule of $M$ such that kerf $\subseteq N$, then $f(N)$ is a weakly 2-absorbing semi-primary submodule of $M$.

Proof. Let $a_{1}, a_{2} \in R$ and $\dot{m} \in M^{\prime}$ such that $0 \neq a_{1} a_{2} \dot{m} \in f(N)$. Thus $0 \neq a_{1} a_{2} \dot{m}_{=}=\dot{m}_{0}$ for some $\dot{m}_{0} \in f(N)$. Since $f$ is an epimorphism, there exist $m \in M$ and $m_{0} \in N$ such that $\dot{m}=f(m)$ and $\dot{m}_{0}=f\left(m_{0}\right)$. This implies that $0 \neq a_{1} a_{2} f(m)=f\left(m_{0}\right)$. Therefore $f\left(a_{1} a_{2} m-m_{0}\right)=0$ and so $a_{1} a_{2} m-m_{0} \in k e r f \subseteq N$. Also, $0 \neq a_{1} a_{2} m \in N$, because if $a_{1} a_{2} m=0$, then $m_{0} \in k e r f$. It follows that $f\left(m_{0}\right)=0$, a contradiction. Now, since $N$ is a weakly 2-absorbing semi-primary, we have $a_{1} a_{2} \in \sqrt{(N: M)}$ or $a_{1} m \in N$ or $a_{2}^{n} m \in N$ for some positive integer $n$. Therefore $a_{1} a_{2} \in \sqrt{\left(f(N): M^{\prime}\right)}$ or $a_{1} \dot{m} \in f(N)$ or $a_{2}^{n} \dot{m} \in f(N)$ for some positive integer $n$. Hence $f(N)$ is a 2-absorbing semi-primary submodule of $M^{\prime}$.

Theorem 2.5. Let $M$ be an $R$-module and $N \subseteq K$ be two submodules of $M$. If $K$ is a weakly 2-absorbing semi-primary submodule of $M$, then $K / N$ is a weakly 2-absorbing semi-primary submodule of $M / N$. 
Proof. Let $a_{1}, a_{2} \in R$ and $m \in M$ such that $N \neq a_{1} a_{2}(m+N) \in(K / N)$. Then $0 \neq a_{1} a_{2} m \in K$. By Definition 2.1, $a_{1} a_{2} \in \sqrt{(K: M)}$ or $a_{1} m \in K$ or $a_{2}^{n} m \in K$ for some positive integer $n$. Therefore $a_{1} a_{2} \in \sqrt{(K / N: M / N)}$ or $a_{1}(m+N) \in K / N$ or $a_{2}^{n}(m+N) \in K / N$ for some positive integer $n$. Hence $K / N$ is a weakly 2-absorbing semi-primary submodule of $M / N$.

Theorem 2.6. Let $M$ be an $R$-module and $N \subseteq K$ be two submodules of $M$. Suppose that $N$ is a weakly 2absorbing semi-primary submodule of $M$. If $K / N$ is a weakly 2-absorbing semi-primary submodule of $M / N$, then $K$ is a weakly 2-absorbing semi-primary submodule of $M$.

Proof. Let $a_{1}, a_{2} \in R$ and $m \in M$ such that $0 \neq a_{1} a_{2} m \in K$. If $a_{1} a_{2} m \in N$, then $0 \neq a_{1} a_{2} m \in N$. By Definition 2.1, $a_{1} a_{2} \in \sqrt{(N: M)} \subseteq \sqrt{(K: M)}$ or $a_{1} m \in N \subseteq K$ or $a_{2}^{n} m \in N \subseteq K$ for some positive integer $n$. If $a_{1} a_{2} m \notin N$, then $N \neq a_{1} a_{2}(m+N) \in N$. Again, by Definition 2.1, $a_{1} a_{2} \in \sqrt{(K / N: M / N)}$ or $a_{1}(m+N) \in K / N$ or $a_{2}^{n}(m+N) \in K / N$ for some positive integer $n$. Thus $a_{1} a_{2} \in \sqrt{(K: M)}$ or $a_{1} m \in K$ or $a_{2}^{n} m \in K$ for some positive integer $n$. Hence $K$ is a weakly 2-absorbing semi-primary submodule of $M$.

Corollary 2.1. Then $N$ is a weakly 2-absorbing semi-primary submodule of an R-module $M$ if and only if $N /\{0\}$ is a weakly 2-absorbing semi-primary submodule of an $R$-module $M /\{0\}$.

Proof. It is straightforward by Theorem 2.5 and Theorem 2.6.

Theorem 2.7. Let $N$ be a submodule of an $R$-module $M$ and $S$ be a multiplicative subset of $R$. If $N$ is a weakly 2-absorbing semi-primary submodule of $M$ such that $(N: M) \cap S=\emptyset$, then $S^{-1} N$ is a weakly 2-absorbing semi-primary submodule of $S^{-1} M$.

Proof. Clearly, $S^{-1} N$ is a proper submodule of $S^{-1} M$. Let $a_{1}, a_{2} \in R, s_{1}, s_{2}, s_{3} \in S$ and $m \in M$ such that $0 \neq \frac{a_{1}}{s_{1}} \frac{a_{2}}{s_{2}} \frac{m}{s_{3}} \in S^{-1} N$. Then there exists $s \in S$ such that $s a_{1} a_{2} m \in N$. If $s a_{1} a_{2} m=0$, then $\frac{a_{1}}{s_{1}} \frac{a_{2}}{s_{2}} \frac{m}{s_{3}}=$ $\frac{s a_{1}}{s s_{1}} \frac{a_{2}}{s_{2}} \frac{m}{s_{3}}=\frac{0}{1}$, a contradiction. If $s a_{1} a_{2} m \neq 0$, then $0 \neq a_{1} a_{2}(s m) \in N$. By Definition $2.1, a_{1} a_{2} \in \sqrt{(N: M)}$ or $a_{1} s m \in N$ or $a_{2}^{n} s m \in N$ for some positive integer $n$. Thus $\frac{a_{1}}{s_{1}} \frac{a_{2}}{s_{2}} \in \sqrt{\left(S^{-1} N: S^{-1} M\right)}$ or $\frac{a_{1}}{s_{1}} \frac{m}{s_{3}}=\frac{a_{1} s m}{s_{1} s_{3} s} \in$ $S^{-1} N$ or $\left(\frac{a_{2}}{s_{2}}\right)^{n} \frac{m}{s_{3}}=\frac{a_{2}^{n} s m}{s_{2}^{n} s_{3} s} \in S^{-1} N$ for some positive integer $n$. Hence $S^{-1} N$ is a weakly 2-absorbing semi-primary submodule of $S^{-1} M$.

Theorem 2.8. Let $N$ be a submodule of an $R$-module $M$ and $S$ be a multiplicative subset of $R$. If $S^{-1} N$ is a weakly 2-absorbing semi-primary submodule of $S^{-1} M$ such that $S \cap Z d(N)=\emptyset$ and $S \cap Z d(M / N)=\emptyset$, then $N$ is a weakly 2-absorbing semi-primary submodule of $M$.

Proof. Let $a_{1}, a_{2} \in R$ and $m \in M$ such that $0 \neq a_{1} a_{2} m \in N$. Then $\frac{a_{1}}{1} \frac{a_{2}}{1} \frac{m}{1} \in S^{-1} N$. If $\frac{a_{1}}{1} \frac{a_{2}}{1} \frac{m}{1}=\frac{0}{1}$, then there exists $s \in S$ such that $s a_{1} a_{2} m=0$ which is a contradiction. If $\frac{a_{1}}{1} \frac{a_{2}}{1} \frac{m}{1} \neq \frac{0}{1}$, then $\frac{0}{1} \neq \frac{a_{1}}{1} \frac{a_{2}}{1} \frac{m}{1} \in S^{-1} N$. By Definition 2.1, $\frac{a_{1}}{1} \frac{a_{2}}{1} \in \sqrt{\left(S^{-1} N: S^{-1} M\right)}$ or $\frac{a_{1}}{1} \frac{m}{1} \in S^{-1} N$ or $\left(\frac{a_{2}}{1}\right)^{n} \frac{m}{1} \in S^{-1} N$ for some positive integer 
$n$. If $\frac{a_{1}}{1} \frac{a_{2}}{1} \in \sqrt{\left(S^{-1} N: S^{-1} M\right)}$, then $\left(\frac{a_{1}}{1} \frac{a_{2}}{1}\right)^{n} \in\left(S^{-1} N: S^{-1} M\right)$ for some positive integer $n$. Thus there exists $s \in S$ such that $s\left(a_{1} a_{2}\right)^{n} M \subseteq N$ for some positive integer $n$. Since $S \cap Z d(M / N)=\emptyset$, we have $\left(a_{1} a_{2}\right)^{n} M \subseteq N$ so $a_{1} a_{2} \in \sqrt{(N: M)}$. If $\frac{a_{1}}{1} \frac{m}{1} \in S^{-1} N$, there exists $s \in S$ such that $s a_{1} m \in N$. Thus $s\left(a_{1} m+N\right)=s a_{1} m+N=N$. But $S \cap Z d(M / N)=\emptyset, a_{1} m \in N$. If $\left(\frac{a_{2}}{1}\right)^{n} \frac{a_{m}}{1} \in N$, there exists $s \in S$ such that such that $s a_{1}^{n} m \in N$ for some positive integer $n$. Thus $s\left(a_{2}^{n} m+N\right)=s a_{2}^{n} m+N=N$ for some positive integer $n$. Since $S \cap Z d(M / N)=\emptyset$, we have $a_{2}^{n} m \in N$ for some positive integer $n$. Therefore $N$ is a weakly 2-absorbing semi-primary submodule of $M$.

Theorem 2.9. Let $N$ be a proper submodule of an $R$-module $M$. The following conditions are equivalent:

(1) $N$ is a weakly 2-absorbing semi-primary submodule of $M$.

(2) For every $a_{1}, a_{2} \in R-(N: M)$ if $a_{1} a_{2} \in R-\sqrt{(N: M)}$, then $\left(N: a_{1} a_{2}\right) \subseteq\left(0: a_{1} a_{2}\right) \cup(N:$ $\left.a_{1}\right) \cup\left(N: a_{2}^{n}\right)$ for some positive integer $n$.

(3) For every $a_{1}, a_{2} \in R-(N: M)$ if $R$ is a u-ring and $a_{1} a_{2} \in R-\sqrt{(N: M)}$, then $\left(N: a_{1} a_{2}\right) \subseteq(0:$ $\left.a_{1} a_{2}\right)$ or $\left(N: a_{1} a_{2}\right) \subseteq\left(N: a_{1}\right)$ or $\left(N: a_{1} a_{2}\right) \subseteq\left(N: a_{2}^{n}\right)$ for some positive integer $n$.

Proof. $(1 \Rightarrow 2)$ Let $m \in\left(N: a_{1} a_{2}\right)$. Then $a_{1} a_{2} m \in N$. If $a_{1} a_{2} m=0$, then $m \in\left(0: a_{1} a_{2}\right) \subseteq(0:$ $\left.a_{1} a_{2}\right) \cup\left(N: a_{1}\right) \cup\left(N: a_{2}^{n}\right)$ for some positive integer $n$. If $a_{1} a_{2} m \neq 0$, then $0 \neq a_{1} a_{2} m \in N$. By Definition 2.1, $a_{1} a_{2} \in \sqrt{(N: M)}$ or $a_{1} m \in N$ or $a_{2}^{n} m \in N$ for some positive integer $n$. But $a_{1} a_{2} \in R-\sqrt{(N: M)}$, $m \in\left(N: a_{1}\right)$ or $m \in\left(N: a_{2}^{n}\right)$ for some positive integer $n$. Therefore $m \in\left(N: a_{1}\right) \cup\left(N: a_{2}^{n}\right)$ for some positive integer $n$. Hence $\left(N: a_{1} a_{2}\right)=\left(0: a_{1} a_{2}\right) \cup\left(N: a_{1}\right) \cup\left(N: a_{2}^{n}\right)$ for some positive integer $n$.

$(2 \Leftrightarrow 3)$ It is obvious.

$(2 \Rightarrow 1)$ Let $a_{1}, a_{2} \in R$ such that $0 \neq a_{1} a_{2} m \in N$. Then $m \in\left(N: a_{1} a_{2}\right)$ and $m \notin(N: 0)$. By assumption, $m \in\left(0: a_{1} a_{2}\right) \cup\left(N: a_{1}\right) \cup\left(N: a_{2}^{n}\right)$ for some positive integer $n$. Clearly, $a_{1} m \in N$ or $a_{2}^{n} m \in N$ for some positive integer $n$. Hence $N$ is a weakly 2-absorbing semi-primary submodule of $M$.

Corollary 2.2. Let $N$ be a proper submodule of an $R$-module $M$. The following conditions are equivalent:

(1) $N$ is a weakly 2-absorbing semi-primary submodule of $M$.

(2) For every $a \in R-(N: M)$ and every ideal $I$ of $R$ such that $I \nsubseteq(N: M)$, if $a I \nsubseteq \sqrt{(N: M)}$, then $(N: a I) \subseteq(0: a I) \cup(N: a) \cup\left(N: I^{n}\right)$ for some positive integer $n$.

(3) For every $a \in R-(N: M)$ and every ideal $I$ of $R$ such that $I \nsubseteq(N: M)$, if $R$ is a u-ring and $a I \nsubseteq \sqrt{(N: M)}$, then $(N: a I) \subseteq(0: a I)$ or $(N: a I) \subseteq(N: a)$ or $(N: a I) \subseteq\left(N: I^{n}\right)$ for some positive integer $n$.

(4) For every ideals $I, J$ of $R$ such that $I, J \nsubseteq(N: M)$, if $I J \nsubseteq \sqrt{(N: M)}$, then $(N: I J) \subseteq(0:$ $I J) \cup(N: I) \cup\left(N: J^{n}\right)$ for some positive integer $n$.

(5) For every ideals $I, J$ of $R$ such that $I, J \nsubseteq(N: M)$, if $R$ is a u-ring and $I J \nsubseteq \sqrt{(N: M)}$, then $(N: I J) \subseteq(0: I J)$ or $(N: I J) \subseteq(N: I)$ or $(N: I J) \subseteq\left(N: J^{n}\right)$ for some positive integer $n$. 
Proof. It is clear from Theorem 2.9 .

Theorem 2.10. Let $N$ be a proper submodule of an $R$-module $M$. The following conditions are equivalent:

(1) $N$ is a weakly 2-absorbing semi-primary submodule of $M$.

(2) For every $a \in R-(N: M)$ and $m \in M$, if am $\notin N$, then $(N:$ am $) \subseteq(0: a m) \cup(\sqrt{((N: M)}$ : a) $\cup \sqrt{(N: m)}$.

Proof. $(1 \Rightarrow 2)$ Let $a \in R-(N: M)$ and $m \in M$ such that am $\notin N$. Assume that $r \in(N: a m)$. Then ram $\in N$. If $r a m \neq 0$, then $0 \neq \operatorname{ram} \in N$. By Definition 2.1, ar $\in \sqrt{(N: M)}$ or $a m \in N$ or $r^{n} m \in N$ for some positive integer $n$. Since $a m \notin N$, we have $r \in(\sqrt{(N: M)}: a)$ or $r \in \sqrt{(N: m)}$. This implies that $r \in(\sqrt{(N: M)}: a) \cup \sqrt{(N: m)} \subseteq(0: a m) \cup(\sqrt{((N: M)}: a) \cup \sqrt{(N: m)}$. Thus $(N: a m) \subseteq(0:$ $a m) \cup(\sqrt{((N: M)}: a) \cup \sqrt{(N: m)}$. If $r a m=0$, then $r \in(0: a m) \subseteq(0: a m) \cup(\sqrt{((N: M)}: a) \cup \sqrt{(N: m)}$. Therefore $(N: a m) \subseteq(0: a m) \cup(\sqrt{((N: M)}: a) \cup \sqrt{(N: m)}$.

$(2 \Rightarrow 1)$ It is clear.

Corollary 2.3. Let $N$ be a proper submodule of an $R$-module $M$. The following conditions are equivalent:

(1) $N$ is a weakly 2-absorbing semi-primary submodule of $M$.

(2) For every ideal $I$ of $R$ such that $I \subseteq R-(N: M)$ and $m \in M$, if $\operatorname{Im} \nsubseteq N$, then $(N: \operatorname{Im}) \subseteq(0$ : Im $) \cup(\sqrt{(N: M)}: I) \cup \sqrt{(N: m)}$.

Proof. It is clear from Theorem 2.10 .

Definition 2.2. Let $N$ be a proper submodule of $M$. If $N$ is a 2-absorbing semi-primary submodule and $a_{1} a_{2} m=0, a_{1} a_{2} \notin \sqrt{(N: M)}, a_{1} m \notin N$ and $a_{2}^{n} m \notin N$ for all positive integer $n$, then $\left(a_{1}, a_{2}, m\right)$ is called $a$ absorbing semi-primary triple-zero of $N$ where $a_{1}, a_{2} \in R, m \in M$.

Theorem 2.11. Let $N$ be a weakly 2-absorbing semi-primary submodule of an $R$-module $M$. Suppose that $K$ is a submodule of $M$ and $a_{1}, a_{2} \in R$ such that $N \subseteq K$ and $a_{1} a_{2} K \subseteq N$. If $\left(a_{1}, a_{2}, m\right)$ is not a absorbing semi-primary triple-zero of $N$ for every $m \in K$, then $a_{1} a_{2} \in \sqrt{(K: M)}$ or $a_{1} K \subseteq N$ or $a_{2}^{n} K \subseteq N$ for some positive integer $n$.

Proof. Assume that $a_{1} a_{2} \notin \sqrt{(K: M)}, a_{1} K \nsubseteq N$ and $a_{2}^{n} K \nsubseteq N$ for all positive integer $n$. Then there are $k_{1}, k_{2} \in K$ such that $a_{1} k_{1} \notin N$ and $a_{2}^{n} k_{2} \notin N$ for all positive integer $n$. If $a_{1} a_{2} k_{1} \neq 0$, then $0 \neq a_{1} a_{2} k_{1} \in N$. By Definition 2.1, $a_{2}^{n_{1}} k_{1} \in N$ for some positive integer $n_{1}$. So let $a_{1} a_{2} k_{1}=0$. By Definition $2.2, a_{2}^{n_{2}} k_{1} \in N$ for some positive integer $n_{2}$. Now if $a_{1} a_{2} k_{2} \neq 0$, then $0 \neq a_{1} a_{2} k_{2} \in N$. Again, by Definition 2.1, $a k_{2} \in N$. Next let $a_{1} a_{2} k_{2}=0$. Now by Definition 2.2, $a_{1} k_{2} \in N$. Let $n_{0}=\max \left\{n_{1}, n_{2}\right\}$. Then $a_{2}^{n_{0}} k_{1}, a_{1} k_{2} \in N$. Since $a_{1} a_{2} K \subseteq N$, we have $a_{1} a_{2}\left(k_{1}+k_{2}\right) \in N$. If $a_{1} a_{2}\left(k_{1}+k_{2}\right) \neq 0$, then $0 \neq a_{1} a_{2}\left(k_{1}+k_{2}\right) \in N$. Thus by Definition 2.1, $a_{1}\left(k_{1}+k_{2}\right) \in N$ or $a_{2}^{n_{3}}\left(k_{1}+k_{2}\right) \in N$ for some positive integer $n_{3}$. This implies that $a_{1} k_{1} \in N$ 
or $a_{2}^{n_{4}} k_{2} \in N$ where $n_{4}=\max \left\{n_{0}, n_{3}\right\}$ and we get a contradiction. Assume that $a_{1} a_{2}\left(k_{1}+k_{2}\right)=0$. New since $\left(a_{1}, a_{2}, k_{1}+k_{2}\right)$ is not a absorbing semi-primary triple-zero of $N$, we have $a_{1}\left(k_{1}+k_{2}\right) \in N$ or $a_{2}^{n_{5}}\left(k_{1}+k_{2}\right) \in N$ for some positive integer $n_{5}$. Clearly, $a_{1} k_{1} \in N$ or $a_{2}^{n_{6}} k_{2} \in N$, where $n_{6}=\max \left\{n_{0}, n_{5}\right\}$, which again is a contradiction. Hence $a_{1} a_{2} \in \sqrt{(K: M)}$ or $a_{1} K \subseteq N$ or $a_{2}^{n} K \subseteq N$ for some positive integer $n$.

Theorem 2.12. Let $N$ be a weakly 2-absorbing semi-primary submodule of an $R$-module $M$. Suppose that $\left(a_{1}, a_{2}, m\right)$ is a absorbing semi-primary triple-zero of $N$ for some $a_{1}, a_{2} \in R$ and $m \in M$. Then

(1) $a_{1} a_{2} N=\{0\}$;

(2) $a_{1}(N: M) m=\{0\}$;

(3) $(N: M) a_{2} m=\{0\}$;

(4) $(N: M)^{2} m=\{0\}$;

(5) $a_{1}(N: M) N=\{0\}$;

(6) $(N: M) a_{2} N=\{0\}$.

Proof. 1. Suppose that $a_{1} a_{2} N \neq\{0\}$. Then there exists $m_{0} \in N$ such that $a_{1} a_{2} m_{0} \notin\{0\}$. Thus $a_{1} a_{2} m+$ $a_{1} a_{2} m_{0} \neq 0$ so $0 \neq a_{1} a_{2}\left(m+m_{0}\right) \in N$. By Definition 2.1, $a_{1} a_{2} \in \sqrt{(N: M)}$ or $a_{1}\left(m+m_{0}\right) \in N$ or $a_{2}^{n}\left(m+m_{0}\right) \in N$ for some positive integer $n$. Therefore $a_{1} a_{2} \in \sqrt{(N: M)}$ or $a_{1} m \in N$ or $a_{2}^{n} m \in N$ for some positive integer $n$. This is a contradiction. Hence $a_{1} a_{2} N=\{0\}$.

2. Suppose that $a_{1}(N: M) m \neq\{0\}$. Then there exists $r \in(N: M)$ such that $a_{1} r m \neq 0$. Since $r m \in N$, we have $0 \neq a_{1}\left(a_{2}+r\right) m \in N$. By Definition 2.1, $a_{1}\left(a_{2}+r\right) \in \sqrt{(N: M)}$ or $a_{1} m \in N$ or $\left(a_{2}+r\right)^{n} m \in N$ for some positive integer $n$. Thus $a_{1} a_{2} \in \sqrt{(N: M)}$ or $a_{1} m \in N$ or $a_{2}^{n} \in N$ for some positive integer $n$. This is a contradiction. Hence $a_{1}(N: M) m=\{0\}$.

3. The proof is similar to part 2 .

4. Assume that $(N: M)^{2} m \neq\{0\}$. Then there exist $r, s \in(N: M)$ such that $r s m \neq 0$. Then by parts 1 and 2, $\left(a_{1}+r\right)\left(a_{2}+s\right) m \neq 0$. Clearly, $0 \neq\left(a_{1}+r\right)\left(a_{2}+s\right) m \in N$. By Definition 2.1, $\left(a_{1}+r\right)\left(a_{2}+s\right) \in \sqrt{(N: M)}$ or $\left(a_{1}+r\right) m \in N$ or $\left(a_{2}+s\right)^{n} m \in N$ for some positive integer $n$. Therefore $a_{1} a_{2} \in \sqrt{(N: M)}$ or $a_{1} m \in N$ or $a_{2}^{n} \in N$ for some positive integer $n$. This is a contradiction. Hence $(N: M)^{2} m=\{0\}$.

5. Suppose that $a_{1}(N: M) N \neq\{0\}$. Then there exist $r \in(N: M)$ and $m_{0} \in N$ such that $a_{1} r m_{0} \neq 0$. Therefore by parts 1 and 2 we conclude that $a_{1}\left(a_{2}+r\right)\left(m+m_{0}\right) \neq 0$. Clearly, $0 \neq a_{1}\left(a_{2}+r\right)\left(m+m_{0}\right) \in N$. By Definition 2.1, $a_{1}\left(a_{2}+r\right) \in \sqrt{(N: M)}$ or $a_{1}\left(m+m_{0}\right) \in N$ or $\left(a_{2}+r\right)^{n}\left(m+m_{0}\right) \in N$ for some positive integer $n$. Therefore $a_{1} a_{2} \in \sqrt{(N: M)}$ or $a_{1} m \in N$ or $a_{2}^{n} m \in N$ for some positive integer $n$. This is a contradiction. Hence $a_{1}(N: M) N=\{0\}$.

6. The proof is similar to part 5. 
Theorem 2.13. Let $M$ be an $R$-module. If $N$ is a weakly 2-absorbing semi-primary submodule of $M$ that is not 2-absorbing semi-primary, then $(N: M)^{2} N=\{0\}$.

Proof. Suppose that $N$ is a weakly 2-absorbing semi-primary submodule of $M$ that is not 2-absorbing semi-primary submodule. Then there exists a absorbing semi-primary triple-zero $\left(a_{1}, a_{2}, m\right)$ of $N$ for some $a_{1}, a_{2} \in R$ and $m \in M$. Assume that $(N: M)^{2} N \neq\{0\}$. Then there exist $r, s \in(N: M)$ and $m_{0} \in N$ such that $r s m_{0} \neq 0$. Since $\left(a_{1}+r\right)\left(a_{2}+s\right)\left(m+m_{0}\right) \neq 0$, we have $0 \neq\left(a_{1}+r\right)\left(a_{2}+s\right)\left(m+m_{0}\right) \in N$. By Definition 2.1, $\left(a_{1}+r\right)\left(a_{2}+s\right) \in \sqrt{(N: M)}$ or $\left(a_{1}+r\right)(m+n) \in N$ or $\left(a_{2}+s\right)^{n}(m+n) \in N$ for some positive integer $n$. Therefore $a_{1} a_{2} \in \sqrt{(N: M)}$ or $a_{1} m \in N$ or $a_{2}^{n} m \in N$. This is a contradiction. Hence $(N: M)^{2} N=\{0\}$.

Corollary 2.4. Let $M$ be a multiplication $R$-module. If $N$ is a weakly 2-absorbing semi-primary submodule of $M$ that is not 2-absorbing semi-primary submodule, then $N^{3}=\{0\}$.

Proof. Suppose that $N$ is a weakly 2-absorbing semi-primary submodule of $M$ that is not 2-absorbing semiprimary submodule. By assumption, $N=(N: M) M$. Then by Theorem $2.13, N^{3}=(N: M)^{3} M=(N:$ $M)^{2}((N: M) M)=(N: M)^{2} N=\{0\}$.

Lemma 2.1. Suppose that $N$ is a weakly 2-absorbing semi-primary submodule of an $R$-module $M$ and $\left(0: m_{2}\right)$ is a 2-absorbing primary ideal of a ring $R$ where $m_{2} \in M-N$. For all $m_{1} \in M$, if $r s \in(N:$ $\left.m_{1}\right)-\sqrt{\left(N: m_{2}\right)}$, then $\left(N: r s m_{2}\right) \subseteq\left(N: r m_{2}\right) \cup \sqrt{\left(N: s^{n} m_{2}\right)}$ for some positive integer $n$.

Proof. Suppose that $r s \in\left(N: m_{1}\right)-\left(N: m_{2}\right)$ where $m_{1} \in M$ and $m_{2} \in M-N$. Let $a \in\left(N: r s m_{2}\right)$. Then $($ ars $) m_{2}=a\left(r s m_{2}\right) \in N$ so ars $\in\left(N: m_{2}\right)$. If $\operatorname{arsm}_{2} \neq 0$, then $0 \neq \operatorname{ars} \in\left(N: m_{2}\right)$. By assumption, ar $\in\left(N: m_{2}\right)$ or $a s \in \sqrt{\left(N: m_{2}\right)}$ or $r s \in \sqrt{\left(N: m_{2}\right)}$. By the assumption, ar $\in\left(N: m_{2}\right)$ or $a s \in \sqrt{\left(N: m_{2}\right)}$. Thus $a \in\left(N: r m_{2}\right)$ or $a \in \sqrt{\left(N: s^{n} m_{2}\right)}$ for some positive integer $n$. This implies that $\left(N: r s m_{2}\right) \subseteq\left(N: r m_{2}\right) \cup \sqrt{\left(N: s^{n} m_{2}\right)}$ for some positive integer $n$. Now if arsm $m_{2}=0$, then ars $\in\left(0: m_{2}\right)$. Thus $a r \in\left(0: m_{2}\right)$ or $a s \in \sqrt{\left(N: m_{2}\right)}$ or $r s \in \sqrt{\left(N: m_{2}\right)}$. Therefore $\left(N: r s m_{2}\right) \subseteq\left(N: r m_{2}\right) \cup \sqrt{\left(N: s^{n} m_{2}\right)}$ for some positive integer $n$.

Proposition 2.1. Let $N$ be an irreducible submodule of an $R$-module $M$. For all $r \in R$ if $(N: r)=\left(N: r^{2}\right)$, then $N$ is a weakly 2-absorbing semi-primary submodule of $M$.

Proof. Let $a_{1}, a_{2} \in R$ and $m \in M$ such that $0 \neq a_{1} a_{2} m \in N$. Suppose that $a_{1} a_{2} \notin \sqrt{(N: M)}, a_{1} m \notin N$ and $a_{2}^{n} m \notin N$ for all positive integer $n$. Clearly, $N \subseteq\left(N+a_{1} a_{2} M\right) \cap\left(N+R a_{1} m\right) \cap\left(N+R a_{2}^{n} m\right)$ for all positive integer $n$. Let $m_{0} \in\left(N+a_{1} a_{2} M\right) \cap\left(N+R a_{1} m\right) \cap\left(N+R a_{2}^{n} m\right)$. This implies that $m_{0} \in N+a_{1} a_{2} M, m_{0} \in$ $N+R a_{1} m$ and $m_{0} \in N+R a_{2}^{n} m$. Then there exist $r_{1}, r_{2} \in R, m_{1} \in M$ and $n_{1}, n_{2} \in N$ such that $n_{1}+a_{1} a_{2} m_{1}=$ $m_{0}=n_{2}+r_{1} a_{1} m=m_{0}=n_{3}+b_{2}^{n} m$. Since $a_{1} n_{1}+a_{1}^{2} a_{2} m_{1}=a_{1} m_{0}=a_{1} n_{2}+r_{1} a_{1}^{2} m=a_{1} m_{0}=a_{1} n_{3}+a_{1} b_{2}^{n} m$, 
we have $a_{1}^{2} r_{1} m \in N$. It follows that $r_{1} m \in\left(N: a_{1}^{2}\right)$. By the assumption, $r_{1} m \in\left(N: a_{1}\right)$, so that $r_{1} a_{1} m \in N$. Thus $N=\left(N+a_{1} a_{2} M\right) \cap\left(N+R a_{1} m\right) \cap\left(N+R a_{2}^{n} m\right)$. Now since $N$ is an irreducible, we have $N+a_{1} a_{2} M \subseteq N$ or $a_{1} m \in N+R a_{1} m \subseteq N$ or $a_{2}^{n} m \in N+R a_{2}^{n} m \subseteq N$, a contradiction. Hence $N$ is a weakly 2-absorbing semi-primary submodule of $M$.

Theorem 2.14. Let $M_{i}$ be an $R_{i}$-module and $N_{i}$ be a proper submodule of $M_{i}$, for $i=1,2$. If $N_{1} \times M_{2}$ is a weakly 2-absorbing semi-primary submodule of $M_{1} \times M_{2}$, then $N_{1}$ is a weakly 2-absorbing semi-primary submodule of $M_{1}$.

Proof. Suppose that $N_{1} \times M_{2}$ is a weakly 2-absorbing semi-primary submodule of $M_{1} \times M_{2}$. Let $a_{1}, a_{2} \in R_{1}$ and $m \in M_{1}$ such that $0 \neq a_{1} a_{2} m \in N_{1}$. Then $(0,0) \neq\left(a_{1}, 0\right)\left(a_{2}, 0\right)(m, 0)=\left(a_{1} a_{2} m, 0\right) \in N_{1} \times M_{2}$. By Definition 2.1, $\left(a_{1} a_{2}, 0\right)=\left(a_{1}, 0\right)\left(a_{2}, 0\right) \in \sqrt{\left(N_{1} \times M_{2}: M_{1} \times M_{2}\right)}$ or $\left(a_{1} m, 0\right)=\left(a_{1}, 0\right)(m, 0) \in N_{1} \times M_{2}$ or $\left(a_{2}^{n} m, 0\right)=\left(a_{2}, 0\right)^{n}(m, 0) \in N_{1} \times M_{2}$ for some positive integer $n$. This implies that $a_{1} a_{2} \in \sqrt{\left(N_{1}: M_{1}\right)}$ or $a_{1} m \in N_{1}$ or $a_{2}^{n} m \in N_{1}$ for some positive integer $n$. Hence $N_{1}$ is a weakly 2-absorbing semi-primary submodule of $M_{1}$.

Corollary 2.5. Let $M_{i}$ be an $R_{i}$-module and $N_{i}$ be a proper submodule of $M_{i}$, for $i=1,2$. If $M_{1} \times N_{2}$ is a weakly 2-absorbing semi-primary submodule of $M_{1} \times M_{2}$, then $N_{2}$ is a weakly 2-absorbing semi-primary submodule of $M_{2}$.

Proof. It is clear from Theorem 2.14.

Corollary 2.6. Let $M_{i}$ be an $R_{i}$-module and $N_{i}$ be a proper submodule of $M_{i}$, for $i=1,2, \ldots, k$. If $M_{1} \times$ $M_{2} \times \ldots \times M_{j-1} \times N_{j} \times M_{j+1} \times \ldots \times M_{k}$ is a weakly 2-absorbing semi-primary submodule of $M_{1} \times M_{2} \times \ldots \times M_{k}$, then $N_{j}$ is a weakly 2-absorbing semi-primary submodule of $M_{j}$.

Proof. It is clear from Theorem 2.14 and Corollary 2.5.

Theorem 2.15. Let $M_{i}$ be an $R$-module and let $N_{i}$ be a proper submodule of $M_{i}$, for $i=1,2$. Then the following conditions are equivalent:

(1) $N_{1} \times M_{2}$ is a weakly 2-absorbing semi-primary submodule of $M_{1} \times M_{2}$.

(2) (a) $N_{1}$ is a weakly 2-absorbing semi-primary submodule of $M_{1}$.

(b) For each $a_{1}, a_{2} \in R$ and $m \in M_{1}$ such that $a_{1} a_{2} m=0$, if $a_{1} a_{2} \notin \sqrt{\left(N_{1}: M_{1}\right)}$ and $a_{1} m \notin$ $N_{1}, a_{2}^{n} m \notin N_{1}$ for all positive integer $n$, then $a_{1} a_{2} \in\left(0: M_{2}\right)$.

Proof. $(1 \Rightarrow 2)$. (a). This follows from Theorem 2.14.

(b). Let $a_{1} a_{2} m=0, a_{1} m \notin N_{1}$ and $a_{2}^{n} m \notin N_{1}$ for all positive integer $n$, where $a_{1}, a_{2} \in R$ and $m \in M_{1}$. Suppose that $a_{1} a_{2} \notin\left(0: M_{2}\right)$. There exists $m_{2} \in M_{2}$ such that $a_{1} a_{2} m_{2} \neq 0$. Thus $(0,0) \neq a_{1} a_{2}\left(m, m_{2}\right)=$ 
$\left(a_{1} a_{2} m, a_{1} a_{2} m_{2}\right) \in N_{1} \times M_{2}$. By part 1, i.e., $a_{1} a_{2} \in \sqrt{\left(N_{1} \times M_{2}: M_{1} \times M_{2}\right)}$ or $a_{1}\left(m, m_{2}\right) \in N_{1} \times M_{2}$ or $a_{2}^{n}\left(m, m_{2}\right) \in N_{1} \times M_{2}$ for some positive integer $n$. Thus $a_{1} a_{2} \in \sqrt{\left(N_{1}: M_{1}\right)}$ or $a_{1} m \in N_{1}$ or $a_{2}^{n} m \in N_{1}$ which is a contradiction. Hence $a_{1} a_{2} \in\left(0: M_{2}\right)$.

$(2 \Rightarrow 1)$. Let $a_{1}, a_{2} \in R$ and $\left(m_{1}, m_{2}\right) \in M_{1} \times M_{2}$ such that $(0,0) \neq\left(a_{1} a_{2} m_{1}, a_{1} a_{2} m_{2}\right)=a_{1} a_{2}\left(m_{1}, m_{2}\right) \in$ $N_{1} \times M_{2}$. If $a_{1} a_{2} m_{1} \neq 0$, then $0 \neq a_{1} a_{2} m_{1} \in N_{1}$. By part (a), $a_{1} a_{2} \in \sqrt{\left(N_{1}: M_{1}\right)}$ or $a_{1} m_{1} \in N_{1}$ or $a_{2}^{n} m_{1} \in N_{1}$ for some positive integer $n$. So $a_{1} a_{2} \in \sqrt{\left(N_{1} \times M_{2}: M_{1} \times M_{2}\right)}$ or $a_{1}\left(m_{1}, m_{2}\right)=\left(a_{1} m_{1}, a_{1} m_{2}\right) \in$ $N_{1} \times M_{2}$ or $a_{2}^{n}\left(m_{1}, m_{2}\right)=\left(a_{2}^{n} m_{1}, a_{2}^{n} m_{2}\right) \in N_{1} \times M_{2}$, and thus we are done. If $a_{1} a_{2} m_{1}=0$, then $a_{1} a_{2} m_{2} \neq 0$. Therefore $a_{1} a_{2} \notin\left(0: M_{2}\right)$. By part (b), $a_{1} a_{2} \in \sqrt{\left(N_{1}: M_{1}\right)}$ or $a_{1} m_{1} \in N_{1}$ or $a_{2}^{n} m_{1} \in N_{1}$ for some positive integer $n$. Thus $a_{1} a_{2} \in \sqrt{\left(N_{1} \times M_{2}: M_{1} \times M_{2}\right)}$ or $a_{1}\left(m_{1}, m_{2}\right) \in N_{1} \times M_{2}$ or $a_{2}^{n}\left(m_{1}, m_{2}\right) \in N_{1} \times M_{2}$. Hence $N_{1} \times M_{2}$ is a weakly 2-absorbing semi-primary submodule of $M_{1} \times M_{2}$.

Corollary 2.7. Let $M_{i}$ be an $R$-module and let $N_{i}$ be a proper submodule of $M_{i}$, for $i=1,2$. Then the following conditions are equivalent:

(1) $M_{1} \times N_{2}$ is a weakly 2-absorbing semi-primary submodule of $M_{1} \times M_{2}$.

(2) (a) $N_{2}$ is a weakly 2-absorbing semi-primary submodule of $M_{2}$.

(b) For each $a_{1}, a_{2} \in R$ and $m \in M_{2}$ such that $a_{1} a_{2} m=0$, if $a_{1} a_{2} \notin \sqrt{\left(N_{2}: M_{2}\right)}, a_{1} m \notin N_{2}$ and $a_{2}^{n} m \notin N_{2}$ for all positive integer $n$, then $a_{1} a_{2} \in\left(0: M_{1}\right)$.

Proof. This follows from Theorem 2.15.

Corollary 2.8. Let $M_{i}$ be an $R$-module and let $N_{i}$ be a proper submodule of $M_{i}$, for $i=1,2, \ldots, k$. Then the following conditions are equivalent:

(1) $M_{1} \times M_{2} \times \ldots \times M_{i-1} \times N_{i} \times M_{i+1} \times M_{k}$ is a weakly 2-absorbing semi-primary submodule of $M_{1} \times M_{2} \times \ldots \times M_{k}$.

(2) (a) $N_{i}$ is a weakly 2-absorbing semi-primary submodule of $M_{i}$.

(b) For each $a_{1}, a_{2} \in R$ and $m \in M_{2}$ such that $a_{1} a_{2} m=0$, if $a_{1} a_{2} \notin \sqrt{\left(N_{2}: M_{2}\right)}, a_{1} m \notin N_{2}$ and $a_{2}^{n} m \notin N_{2}$ for all positive integer $n$, then there exists $j \in\{1,2, \ldots, k\}$ such that $a_{1} a_{2} \in\left(0: M_{j}\right)$.

Proof. This follows from Theorem 2.15.

Theorem 2.16. Let $N_{i}$ be a proper submodule of an $R_{i}$-module $M_{i}$, for $i=1,2$. Then the following conditions are equivalent:

(1) $N_{1}$ is a 2-absorbing semi-primary submodule of $M_{1}$.

(2) $N_{1} \times M_{2}$ is a 2-absorbing semi-primary submodule of $M_{1} \times M_{2}$.

(3) $N_{1} \times M_{2}$ is a weakly 2-absorbing semi-primary submodule of $M_{1} \times M_{2}$, where $M_{2} \neq\{0\}$.

Proof. $(1 \Rightarrow 2)$. This is clear, by Theorem 2.15 . 
$(2 \Rightarrow 3)$. The proof is clear.

$(3 \Rightarrow 1)$. Suppose that $N_{1} \times M_{2}$ is a weakly 2-absorbing semi-primary submodule of $M_{1} \times M_{2}$, where $M_{2} \neq\{0\}$. Let $a_{1}, a_{2} \in R_{1}$ and $m \in M_{1}$ such that $a_{1} a_{2} m \in N_{1}$. By assumption, there exists $m_{2} \in M_{2}$ such that $m_{2} \neq 0$. Since $\left(a_{1}, 1\right)\left(a_{2}, 1\right)\left(m, m_{2}\right)=\left(a_{1} a_{2} m, m_{2}\right) \neq(0,0)$, we have $(0,0) \neq\left(a_{1}, 1\right)\left(a_{2}, 1\right)\left(m, m_{2}\right) \in$ $N_{1} \times M_{2}$. By Definition 2.1, $\left(a_{1}, 1\right)\left(a_{2}, 1\right) \in \sqrt{\left(N_{1} \times M_{2}: M_{1} \times M_{2}\right)}$ or $\left(a_{1}, 1\right)\left(m, m_{2}\right) \in N_{1} \times M_{2}$ or $\left(a_{2}, 1\right)^{n}\left(m, m_{2}\right) \in N_{1} \times M_{2}$ for some positive integer $n$. Therefore $a_{1} a_{2} \in \sqrt{\left(N_{1}: M_{1}\right)}$ or $a_{1} m \in N_{1}$ or $a_{2}^{n} m \in N_{1}$ for some positive integer $n$ and hence $N_{1}$ is a 2-absorbing semi-primary submodule of $M_{1}$.

Corollary 2.9. Let $N_{i}$ be a proper submodule of an $R_{i}$-module $M_{i}$, for $i=1,2$. Then the following conditions are equivalent:

(1) $N_{2}$ is a 2-absorbing semi-primary submodule of $M_{1}$.

(2) $M_{1} \times N_{2}$ is a 2-absorbing semi-primary submodule of $M_{1} \times M_{2}$.

(3) $M_{1} \times N_{2}$ is a weakly 2-absorbing semi-primary submodule of $M_{1} \times M_{2}$, where $M_{1} \neq\{0\}$.

Proof. This follows from Theorem 2.16.

Corollary 2.10. Let $N_{i}$ be a proper submodule of an $R_{i}$-module $M_{i}$, for $i=1,2, \ldots, k$. Then the following conditions are equivalent:

(1) $N_{i}$ is a 2-absorbing semi-primary submodule of $M_{1}$.

(2) $M_{1} \times M_{2} \times \ldots \times M_{i-1} \times N_{i} \times M_{i+1} \times M_{k}$ is a 2-absorbing semi-primary submodule of $M_{1} \times M_{2} \times \ldots \times M_{k}$.

(3) $M_{1} \times M_{2} \times \ldots \times M_{i-1} \times N_{i} \times M_{i+1} \times M_{k}$ is a weakly 2-absorbing semi-primary submodule of $M_{1} \times M_{2} \times \ldots \times M_{k}$, where $M_{j} \neq\{0\}$.

Proof. This follows from Theorem 2.16 and Corollary 2.9.

Theorem 2.17. Let $N_{i}$ be a proper submodule of an $R_{i}$-module $M_{i}$, for $i=1,2$. If $N_{1} \times N_{2}$ is a weakly 2-absorbing semi-primary submodule of $M_{1} \times M_{2}$, then

(1) $N_{1}$ is a weakly 2-absorbing semi-primary submodule of $M_{1}$.

(2) $N_{2}$ is a weakly 2-absorbing semi-primary submodule of $M_{2}$.

Proof. (1). Suppose that $N_{1} \times N_{2}$ is a weakly 2-absorbing semi-primary submodule of $M_{1} \times M_{2}$. Let $a_{1}, a_{2} \in$ $R_{1}$ and $m \in M_{1}$ such that $0 \neq a_{1} a_{2} m \in N_{1}$. Clearly, $(0,0) \neq\left(a_{1}, 1\right)\left(a_{2}, 1\right)\left(m, m_{2}\right)=\left(a_{1} a_{2} m, m_{2}\right) \in N_{1} \times N_{2}$. By Definition 2.1, $\left.\left(a_{1} a_{2}, 1\right)=\left(a_{1}, 1\right)\left(a_{2}, 1\right) \in \sqrt{\left(N_{1} \times N_{2}: M_{1} \times M_{2}\right.}\right)$ or $\left(a_{1} m, m_{2}\right)=\left(a_{1}, 1\right)\left(m, m_{2}\right) \in$ $N_{1} \times N_{2}$ or $\left(a_{2}^{n} m, m_{2}\right)=\left(a_{2}, 1\right)^{n}\left(m, m_{2}\right) \in N_{1} \times N_{2}$ for some positive integer $n$. Therefore $a_{1} a_{2} \in \sqrt{\left(N_{1}: M_{1}\right)}$ or $a_{1} m \in N_{1}$ or $a_{2}^{n} m \in N_{1}$ for some positive integer $n$. Hence $N_{1}$ is a weakly 2-absorbing semi-primary submodule of $M_{1}$.

(2). This follows from part 1. 
Example 2.3. Let $M=\boldsymbol{Z} \times \boldsymbol{Z}$ be an $\boldsymbol{Z}$-module. Consider the submodule $N=5 \boldsymbol{Z} \times 12 \boldsymbol{Z}$ of $M$. It is easy to see that $5 \boldsymbol{Z}$ and $12 \boldsymbol{Z}$ are weakly 2-absorbing semi-primary submodule of $M$. Notice that $(0,0) \neq 2 \cdot 3(5,2) \in N$, but $2 \cdot 3 \notin \sqrt{(M: N)}, 2(5,2) \notin N$, and $(2 \cdot 3)^{n} \notin(N: M)$ for all positive integer $n$. Therefore $N$ is not a weakly 2-absorbing semi-primary submodule of $M$. This example shows that the converse of Theorem 2.17 is not true.

Theorem 2.18. Let $N_{i}$ be a submodule of an $R_{i}$-module $M_{i}$, for $i=1,2,3$. If $N$ is a weakly 2 -absorbing semi-primary submodule of $M_{1} \times M_{2} \times M_{3}$, then $N=\{(0,0,0)\}$ or $N$ is a 2-absorbing semi-primary submodule of $M_{1} \times M_{2} \times M_{3}$.

Proof. Suppose that $N$ is a weakly 2-absorbing semi-primary submodule of $M_{1} \times M_{2} \times M_{3}$ that is not 2-absorbing semi-primary. We will show that $N=\{(0,0,0)\}$. Now suppose that $N_{1} \times N_{2} \times N_{3}=N \neq$ $\{0\} \times\{0\} \times\{0\}$. Thus $N_{i} \neq\{0\}$, for some $i=1,2,3$. We claim that $N_{1} \neq\{0\}$. There exists $m_{1} \in N_{1}$ such that $m_{1} \neq 0$. To show that $N_{2}=M_{2}$ or $N_{3}=M_{3}$. Assume that $N_{2} \neq M_{2}$ and $N_{3} \neq M_{3}$. Thus there exist $m_{2} \in$ $M_{2}$ and $m_{3} \in M_{3}$ such that $m_{2} \notin N_{2}$ and $m_{3} \notin N_{3}$. Since $(1,0,1)(1,1,0)\left(m_{1}, m_{2}, m_{3}\right)=\left(m_{1}, 0,0\right) \neq(0,0,0)$, we have $(0,0,0) \neq(1,0,1)(1,1,0)\left(m_{1}, m_{2}, m_{3}\right) \in N_{1} \times N_{2} \times N_{3}$. By Definition 2.1, we get $(1,0,1)(1,1,0) \in$ $\sqrt{\left(N_{1} \times N_{2} \times N_{3}: M_{1} \times M_{2} \times M_{3}\right)}$ or $(1,0,1)\left(m_{1}, m_{2}, m_{3}\right) \in N$ or $(1,1,0)^{n}\left(m_{1}, m_{2}, m_{3}\right) \in N$, for some positive integer $n$. So $m_{2} \in N_{2}$ or $m_{3} \in N_{3}$, a contradiction. Therefore $N=N_{1} \times M_{2} \times N_{3}$ or $N=$ $N_{1} \times N_{2} \times M_{3}$. If $N=N_{1} \times M_{2} \times N_{3}$, then $(0,1,0) \in\left(N: M_{1} \times M_{2} \times M_{3}\right)$. By Theorem $2.13,\{0\} \times M_{2} \times\{0\}=$ $(0,1,0)^{2} N \subseteq\left(N: N_{1} \times M_{2} \times N_{3}\right)^{2} N=\{(0,0,0)\}$, which is a contradiction. Hence $N=\{(0,0,0)\}$.

Theorem 2.19. Let $N_{i}$ be a submodule of an $R_{i}$-module $M_{i}$, for $i=1,2,3$. If $N \neq\{(0,0,0)\}$ and $N$ is a 2-absorbing semi-primary submodule of $M_{1} \times M_{2} \times M_{3}$, then $N$ is a weakly 2-absorbing semi-primary submodule of $M_{1} \times M_{2} \times M_{3}$.

Proof. Similar to the proof of Theorem 2.18

The above theorem shows the relationship between 2-absorbing semi-primary and weakly 2-absorbing semi-primary submodules in $R_{1} \times R_{2} \times R_{3}$-modules. From the above theorem, we have the following corollary.

Corollary 2.11. Let $N_{i}$ be a submodule of an $R_{i}$-module $M_{i}$, for $i=1,2,3$ with $N \neq\{(0,0,0)\}$. Then $N$ is a weakly 2-absorbing semi-primary submodule of $M_{1} \times M_{2} \times M_{3}$ if and only if $N$ is a weakly 2-absorbing semi-primary submodule of $M_{1} \times M_{2} \times M_{3}$.

Proof. This follows from Theorem 2.18.

Corollary 2.12. Let $N_{i}$ be a submodule of an $R_{i}$-module $M_{i}$, for $i=1,2, \ldots, k \geq 3$ with $N \neq\{(0,0, \ldots, 0)\}$. Then $N$ is a weakly 2-absorbing semi-primary submodule of $M_{1} \times M_{2} \times \ldots \times M_{k}$ if and only if $N$ is a weakly 2-absorbing semi-primary submodule of $M_{1} \times M_{2} \times \ldots \times M_{k}$. 
Proof. This follows from Theorem 2.19 .

\section{REFERENCES}

[1] D. Anderson and E. Smith, Weakly prime ideals. Houston J. Math., 29 (2003), 831 - 840.

[2] S.E. Atani and F. Farzalipour, On weakly primary ideals. Georgian Math. J., 12 (3) (2005), 423 - 429.

[3] A. Badawi and A.Y. Darani, On weakly 2-absorbing ideals of commutative rings. Houston J. Math., 39 (2013), 441 - 452.

[4] A. Badawi, U. Tekir and E. Yetkin, On weakly 2-absorbing primary ideals of commutative rings. J. Korean Math. Soc., $52(1)(2015), 97-111$.

[5] M. Behboodi and H. Koohy, Weakly prime modules. Vietnam J. Math., 322 (2004), 185 - 195.

[6] A.Y. Darani and F. Soheilnia, On 2-absorbing and weakly 2- absorbing submodules. Thai J. Math., 9, (2011), 577 - 584.

[7] Z.A. El-Bast and P.F. Smith, Multiplication modules. Comm. Algebra, 6(4)(1988), 755 - 779.

[8] M. Larsen and P. McCarthy, Multiplicative theory of ideals. Academic Press, New York, London (1971).

[9] H. Mostafanasab, On weakly classical primary submodules. Bull. Belg. Math. Soc., 22(5)(2015), 743 - 760.

[10] H. Mostafanasab, U. Tekir and K.H. Oral, Weakly classical prime submodules. Math. J., 56 (2016), 1085 - 1101.

[11] H. Mostafanasab, E. Yetkin, U. Tekir and A.Y. Darani, On 2-absorbing primary submodules of modules over commutative rings. An. St. Univ. Ovidius Constanta, 24(1)(2016), 335 - 351.

[12] Sh. Payrovi and S. Babaei, On the 2-absorbing submodules. Iran. J. Math. Sci. Inform., 10(1) (2015), 131 -137.

[13] R. Sharp, Steps in commutative algebra. Cambridge University Press, Cambridge-New York-Sydney (2000).

[14] N. Zamani, $\phi$-prime submodules. Glasgow Math. J., 52(2) (2010), 253 - 259. 